\title{
EL DILEMA DE LA POLÍTICA ECONÓMICA EN HONDURAS PARA RESOLVER EL ALTO NIVEL DE DESEMPLEO $^{1}$
}

\author{
José Evelio Serrano Tobías² \\ Universidad Tecnológica Centroamericana, Tegucigalpa, Honduras. \\ (Enviado: Agosto, 2016; Aceptado para publicación: Diciembre, 2016)
}

\begin{abstract}
Resumen:
La causa del alto nivel de desempleo en Honduras es la deficiente demanda agregada, que afecta el crecimiento económico del país y conduce a un proceso deflacionario. Para resolver este problema y generar ingreso para amplios sectores de la población, se requiere que el PIB real crezca entre el 6\% y $8 \%$ anual en forma sostenible, estable y equitativa, por un periodo de diez años consecutivos.
\end{abstract}

La solución de este problema está en el Modelo Keynesiano, estimulando la demanda agregada, a través de política económica expansiva, bajando impuestos y tasas de interés, aumentando el gasto público en inversión y devaluando el Lempira. Sin embargo, el contexto económico actual de la economía hondureña hace que, aunque se tengan los instrumentos, no puedan ser aplicados, porque primero hay que resolver los grandes desequilibrios macroeconómicos causados por el exagerado gasto público corriente y la corrupción.

Actualmente, y con el acompañamiento del FMI, se está aplicando una política económica contractiva de corte neoliberal, basada en políticas que estimulan la oferta agregada en vez de la demanda. Lo paradójico es que las políticas contractivas que se están adoptando en vez de reducir el desempleo, lo están aumentando, con lo cual se agrava el problema. Pero, el gran dilema de la política económica hondureña, es que, en el corto plazo, no hay otra opción. Tan pronto como se lograra estabilizar la economía, se podría aplicar el Modelo Keynesiano basado en políticas expansivas, que sí favorecerían una disminución en el desempleo.

Palabras Clave: Dilema, Desempleo, Deflación, Keynesianismo, Neoliberalismo

\begin{abstract}
:
The cause of the high level of unemployment in Honduras is the deficiency in aggregate demand, which affects the economic growth of the country, and at the same time leads to a deflationary process.

The solution to this problem is found in the application of the Keynesian Model by stimulating aggregate demand, through an expansive economic policy, lowering taxes and interest rates, increasing public investment expenditure and devaluing the national currency. However, the current economic context of Honduran economy
\end{abstract}

\footnotetext{
${ }^{1}$ Este Artículo está basado en tres obras fundamentales referentes: 1. Las conferencias del Dr. Julián Pavón de CEPADE, "Muerte y Resurrección de Keynes" de la Universidad Politécnica de Madrid, 2013; 2) Las conferencias sobre "El papel de la política monetaria en México" y "La inflación, recesión y política" de Manuel Cavazos Lerma, CEMLA, 1979; y, 3) La conferencia sobre "Crisis financiera y desorden mundial" del Señor Julio Anguita, miembro del Partido Socialista Español, 2011.Mi agradecimiento sincero es para el Licenciado Edwin Araque Bonilla, Ex presidente del BCH y profesor de Postgrado de UNITEC, por haber revisado el borrador de este artículo y por sus acertados comentarios, a efecto de que el escrito reúna las condiciones de calidad técnico-científicas para su publicación.

${ }^{2}$ Autor para correspondencia. Email: eserrano50@ hotmail.com
} 
does not allow applying these instruments, because, first, it is necessary to solve the great macroeconomic imbalances caused by the excessive current public expenditure and corruption.

Currently, the contractive economic policy being implemented - with the support of the IMF - is based on the neoliberal model, stimulating the aggregate supply but not the aggregate demand. The paradox is that the contractionary policies that are being implemented, rather than contributing to the reduction of unemployment are increasing it, worsening the problem. But the great dilemma of the Honduran economic policy is that, in the short term, there is no choice. As soon as the economy stabilizes, the Keynesian Model could be applied, based on expansionary policies that would favor a drastic decrease in the unemployment rate.

Keywords: Dilemma, Unemployment, Deflation, Keynesianism, Neoliberalism.

\section{Introducción}

La ruta del desarrollo económico y social de un país no es fácil, ya que en su proceso hay que vencer muchos escollos y aplicar una política económica eficaz y eficiente para obtener los resultados esperados. Resulta obvio que los países de la región centroamericana requieren de un crecimiento económico robusto para salir de la pobreza en que se encuentran postrados; pero, no siempre se hace un análisis o se entiende a cabalidad las razones del lento proceso de crecimiento, el cual no sólo depende de la capacidad de sus gobernantes para entender lo que hay que hacer en materia económica, sino también del tipo de modelo económico practicado por los países; así como las imposiciones de los países poderosos sobre los países pobres y las características "endógenas" de las propias economías; siendo, muchas veces, estas tres últimas las causales más importantes.

Centroamérica y Honduras no son la excepción, ya que uno de los graves problemas que enfrentan estos países es la pobreza generalizada, que cada día es mayor por la falta de un empleo digno que garantice cierto nivel de ingreso para la población que les permita mejores niveles de bienestar; pero, actualmente, sólo se alberga la esperanza de que algún día esto se revierta. Por eso, el objetivo fundamental de este trabajo, es analizar las opciones que existen desde la perspectiva de un cambio de modelo económico para revertir esta situación; y, al mismo tiempo, concientizar a las autoridades económicas de que la solución al alto desempleo podría estar en la adopción del Modelo Keynesiano; pero, que su aplicación se ve obstaculizada, por la necesidad de estabilizar la economía de los graves desequilibrios que enfrenta.

Es importante reconocer que la forma para reducir los altos niveles de pobreza es a través del crecimiento económico sostenido, estable y con equidad, a una tasa anual mínima del $6 \%{ }^{3}$

\footnotetext{
${ }^{3}$ El Representante del Banco Mundial en Honduras, Giorgio Valentini, señaló recientemente que "el país enfrenta grandes retos para salir de los altos niveles de pobreza, desempleo y la debilidad institucional, que no permiten cruzar la línea del subdesarrollo". Agregó, además, que "el país requiere de un crecimiento de 5\% ó $6 \%$ anual para lograr tener una economía sostenible". No obstante, en los últimos años, se ha venido sosteniendo que la tasa de crecimiento óptima para los países pobres debe ser del $8 \%$.
} 
por diez años consecutivos, y significativamente superior a la tasa de crecimiento poblacional, ya que sin crecimiento no hay desarrollo.

El crecimiento es una condición sine qua non para el desarrollo y, para crecer se necesita poner en marcha una política económica que contribuya a incrementar la riqueza nacional, favoreciendo el consumo de productos nacionales, la inversión privada productiva, la inversión pública y mejorando la estructura productiva del país, la cual permita producir una mayor cantidad y variedad de productos para el mercado nacional e internacional, de tal manera que se genere el ingreso de divisas apropiado para no tener que estar devaluando la moneda, lo cual constituye una forma artificial y poco efectiva de estimular las exportaciones. Esto tendría un impacto profundo en la calidad de vida de la sociedad hondureña.

Las cifras del Banco Central de Honduras ponen de manifiesto que el crecimiento promedio de la economía hondureña en los últimos 10 años ronda el 3.5\% anual. Además, con base en información del INE, se estima que el crecimiento poblacional promedio del mismo período es de $2.5 \%{ }^{4}$, con lo cual, la brecha entre ambos crecimientos es de apenas el $1.0 \%$. Con este panorama de bajo crecimiento económico y alto crecimiento poblacional, Honduras no saldría del subdesarrollo en el que se encuentra ni en 100 años. De ahí, la gran responsabilidad de los gobiernos de turno, de la sociedad civil, de las universidades, de las iglesias, de los sindicatos y de toda la sociedad hondureña, de trabajar arduamente para lograr dar un salto cualitativo y cuantitativo en el desarrollo del país, para tener en un plazo de sólo 20 años, un mejor país para vivir.

\section{Marco Teórico}

John Maynard Keynes nació en 1883 y en 1936 escribió la obra titulada "La Teoría general del empleo, el interés y el dinero" con la cual da una respuesta categórica a la Gran Depresión de los años 30's. con una propuesta de modelo para corregir el desempleo y la deflación, que eran los dos graves problemas que enfrentaba la economía mundial en ese momento.

\subsection{El Modelo Keynesiano}

Keynes dio aportes fundamentales a la política económica, desde una perspectiva capitalista, para resolver los dos problemas más importantes que afronta comúnmente toda economía, que son el desempleo y la inflación y, para corregir estos problemas, él propuso lo siguiente:

$$
\text { Demanda Agregada: } \mathbf{D g}=\mathbf{C}+\mathbf{I}+\mathbf{G}+\mathbf{X} \text {. }
$$

En donde: $\mathrm{Dg}$ = Demanda global; $\mathrm{C}=$ Demanda para Consumo; $\mathrm{I}=$ Demanda para Inversión; $\mathrm{G}=$ Demanda para Gasto en Inversión del Gobierno; $\mathrm{X}=$ Demanda Externa (Exportaciones). En esta ecuación las importaciones se restan porque son parte de la oferta agregada.

\footnotetext{
${ }^{4}$ Según el último Censo de Población del INE, la tasa de crecimiento poblacional es de 1.99\%.
} 
Para corregir el desempleo y la deflación (que eran los dos graves problemas de la economía mundial en la Gran Depresión) la solución propuesta por Keynes fue la de aumentar la demanda agregada. Y, para eso, había que usar política económica expansiva, bajando los impuestos directos para que la gente tuviera más dinero para gastar y, así, aumentar el consumo; bajar las tasas de interés para que los inversionistas demandaran más crédito para invertir y generar empleo; incrementar el gasto en inversión del gobierno para generar fuentes de empleo e ingreso; devaluar la moneda para hacer más competitiva la economía (Pavón, 2012).

Otro problema que las economías sufren comúnmente es la inflación. Keynes también propuso los instrumentos de política que pueden ayudar a reducir o a eliminar la inflación. Keynes señala, como mecanismo para reducir los precios, bajar la demanda agregada, para lo cual habría que usar una política económica contractiva, que implicaría subir los impuestos directos, subir los tipos de interés, bajar el gasto público, y revaluar la moneda.

Esto es todo lo contrario a las políticas de estímulo de la demanda agregada para eliminar el desempleo y la deflación y, por esa razón, Keynes concluyó que el desempleo y la inflación no pueden coexistir, porque si el problema del desempleo es por deficiencia de demanda agregada, los precios entonces tenderían a la baja y no a subir, dándose entonces el fenómeno de desempleo con deflación, siendo ésta la fórmula perfecta para la aplicación de la Teoría Keynesiana (Pavón, 2012).

La lógica de este planteamiento es que, si hay un exceso de demanda agregada, dicho exceso anula la oferta agregada y ésta, a su vez, incide en un bajo nivel de empleo de mano de obra. Por eso, no puede haber desempleo con inflación. Si hay inflación, el desempleo se reduce o vice-versa. Dicho de otra manera, el gobierno puede elegir entre tener más inflación y menos desempleo, o menos inflación y más desempleo. Esto es lo que tratan las Curvas de Phillips.

\subsection{Las Curvas de Phillips}

Las Curvas de Phillips plantean que, en el corto plazo, a mayor inflación hay menor desempleo o, simplemente, a mayor inyección de dinero en la economía, por parte del Banco Central, mayor inflación y menor desempleo. Quiere decir que, el hacedor de política económica puede decidir inflar la economía para reducir el desempleo. Pero, esto sólo es verdad en el corto plazo, ya que, en el mediano y largo plazo, la verdadera elección no es tener más inflación a cambio de menos desempleo, sino tener más inflación hoy a cambio de más desempleo mañana, porque la solución planteada por las Curvas de Phillips es válida sólo para el corto plazo. Eso provoca desplazamientos en las curvas de Phillips inicial y de corto plazo, ya que en el mediano y largo plazo, por el ajuste de expectativas de los trabajadores y empresarios ${ }^{5}$, la inflación va a converger con la tasa de desempleo natural, y

\footnotetext{
${ }^{5}$ Según Cavazos Lerma, el desempleo se reduce si las expectativas de los trabajadores son diferentes de la inflación actual, ya que si los trabajadores anticiparan perfectamente la inflación esperada (Пe) el desempleo no se reduciría. Por eso, en cuanto mayor sea la diferencia entre la inflación esperada y la inflación actual,
} 
se va a convertir en una línea vertical. Significa que, por más que se infle la economía, el desempleo no se va a reducir (Cavazos Lerma, 1979).

A eso hay que agregar el objetivo cambiario del Banco Central: "dentro del sistema de tipos de cambio fijo con variaciones dentro de la banda de flotación, eso constituye otra limitante, porque la inflación interna no puede ser indefinidamente mayor a la externa" (Cavazos Lerma, 1979). Por lo que, si se infla la economía tratando de reducir el desempleo y se quiere mantener el tipo de cambio dentro de la banda de flotación, tarde o temprano, la inflación interna tendrá que bajar para ajustarse a la externa. Esto producirá lo que se conoce como el temido fenómeno de "Estanflación” (Cavazos Lerma, 1979), combinación inexistente para Keynes.

Al ajustarse en el mediano y largo plazo, la Curva de Phillips de largo plazo se convierte en una línea vertical sobre NAIRU, que es un término que significa "Non-Accelerating Inflation Rate of Unemployment" o, simplemente, Tasa Natural de Desempleo ${ }^{6}$. Esto sucede debido al ajuste de las expectativas de trabajadores y empresarios, que hacen que, por más que se aumente la inflación, el desempleo no disminuirá.

La lección que se puede aprender de las Curvas de Phillips es que la política monetaria (aumentos autónomos en la cantidad de dinero por parte del Banco Central) no puede afectar variables reales, como el desempleo y la producción, más que en el corto plazo. En otras palabras, la política monetaria no puede afectar variables reales en forma permanente (Cavazos Lerma, 1979). Además, existe otro momento en el que la política monetaria se vuelve inoperante, específicamente las tasas de interés, y es ante el fenómeno de la llamada “Trampa de Liquidez". En este caso, la política monetaria pierde su eficacia como instrumento de expansión de la demanda agregada.

A continuación, en la Figura 1, se presenta una ilustración de las Curvas de Phillips de corto y de largo plazo:

mayor será la caída en el desempleo y la razón es porque se origina una baja en el salario real que hace que los empresarios se vean estimulados a emplear más mano de obra, porque les es más barato; pero, a medida que la inflación esperada se acerque a la real o se iguale, el atractivo para emplear más trabajadores desaparecerá y obligadamente habrá inflación y desempleo.

${ }^{6}$ La "Tasa Natural de Desempleo", según Cavazos Lerma, es aquella que es consistente con la estructura de salarios reales de equilibrio. Pero, es importante distinguir que, aunque los salario sean de equilibrio, siempre habrá gente desempleada por rigideces del mercado laboral, tales como insuficiente información respecto a vacantes; por imperfecciones en el mercado de trabajo; por falta de habilidades para cubrir las vacantes que existen o, incluso, porque habrá gente que sencillamente no quiere trabajar. 


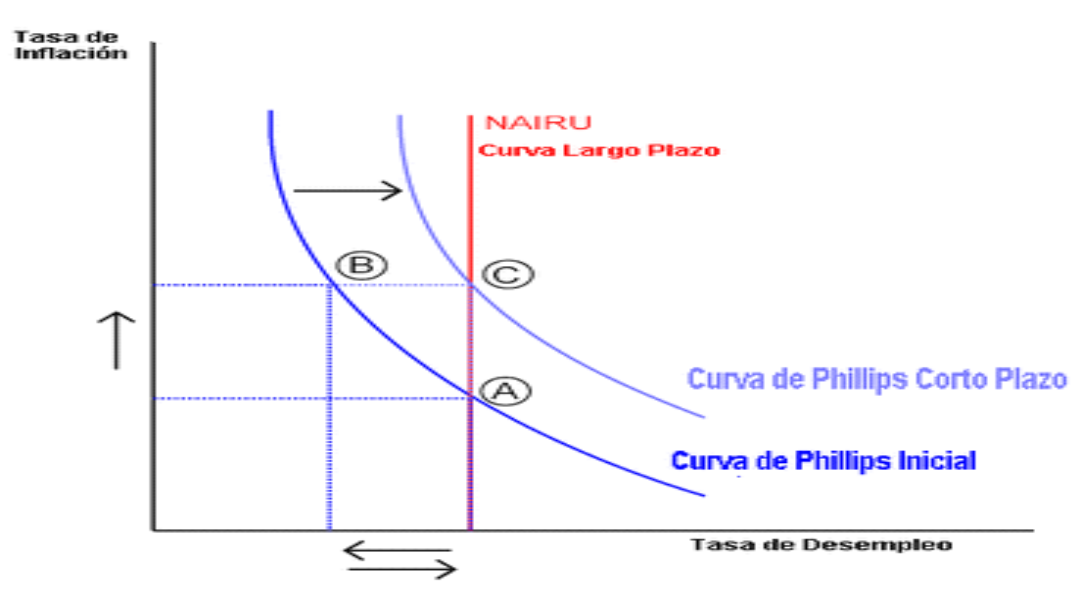

Figura 1. Curvas de Phillips

Fuente: www.google.hn/search?q=curvas + de + phillips \&biw $=931 \&$ bih=590\&tbm=isch\&imgil

\subsection{Los Límites del Modelo Keynesiano}

Como ya se mencionó antes, para resolver el problema de los años 30`s, la solución propuesta por Keynes fue la de aumentar la demanda agregada a través de una política económica expansiva en lo monetario y fiscal, dentro de lo cual se incluye la devaluación de la moneda para hacer más competitivas las exportaciones, ya que al devaluar se obtienen dos efectos: por una parte, se abaratan los bienes exportables a los compradores extranjeros; y, por otra, los exportadores locales reciben más moneda local por cada dólar exportado, lo cual constituye un estímulo para ellos.

Por el lado de las importaciones, hay un desestimulo, ya que éstas se encarecen y por eso les resulta menos atractivo a los agentes económicos demandar dólares. Pero, de nuevo, esto sólo es válido en el corto plazo, porque en el mediano y largo plazo, el mercado se ajustará a su punto de equilibrio a mayores costos y el beneficio desaparecerá permaneciendo únicamente los efectos adversos.

Pero, la teoría keynesiana funcionó desde mediados de los años 30’s hasta principios de la década de los 70, ya que en 1973 ocurre otra crisis la "crisis petrolera" por la cual el precio del petróleo pasó de valer US\$2 el barril a un valor superior a los US\$35, lo que provocó un proceso inflacionario mundial (Pavón, 2012); pero, la diferencia en este caso es que la inflación no es por exceso de demanda agregada, sino por incremento de costos. Sucede, entonces, un fenómeno que nunca antes había ocurrido en la historia económica que es la coexistencia de desempleo con inflación y hasta aquí, como bien señala Pavón, la Teoría Keynesiana ya no llegó (Pavón, 2012).

En esta "coexistencia" los mayores costos van halando a los precios y con una situación de recesión económica los precios siguen subiendo. Entonces se ha trasformado la inflación por demanda en inflación por costos y coexisten el desempleo y la inflación y las políticas 
keynesianas ya no funcionan, por lo cual son abandonadas y sustituidas por las políticas liberales, cuyo abanderado fue Milton Friedman de la Escuela de Chicago.

Con Milton Friedman se inicia el uso de las llamadas políticas de oferta global (ya no son políticas de demanda global como Keynes) y, estas políticas, consideran que el presupuesto público debe ser neutral $(\mathrm{Y}=\mathrm{G})$; que sólo debe manejarse la tasa de interés como instrumento de política monetaria; y que se debe pasar de la macroeconomía a la microeconomía; es decir, la situación propuesta era que si se iban a combatir los altos costos de producción, éstos sólo se pueden combatir desde la empresa misma, a través de: a) bajar los salarios o congelarlos; y, b) bajar los costos de producción a través de la innovación, de la mejora en la calidad, del aumento en la competitividad y la productividad (Pavón, 2012).

Pero este enfoque, si bien ha contribuido a mejorar la situación de las empresas (especialmente las grandes empresas), plantea una situación de franca desventaja para los trabajadores, porque se trata de reducir el número de trabajadores por empresa, lo cual ha contribuido al aumento del desempleo; a reducir el nivel de ingreso y de bienestar de la sociedad; y a acentuar más la pobreza - especialmente en los países del tercer mundo - al tiempo que ha incidido en una mayor concentración del ingreso y de la riqueza. Con este enfoque, el señor Milton Friedman se convierte en el referente indiscutible y se le dice adiós al keynesianismo.

Sin embargo, en el 2008, surge la Crisis Financiera Internacional originada en el mercado hipotecario de los Estados Unidos, cuya situación es similar a la ocurrida en la Gran Depresión de los años 30’s, en donde ocurre recesión y deflación, siendo aquí donde el Modelo Keynesiano cobra vida nuevamente (Pavón, 2012). Esto podría explicar la enorme inyección de dinero del Gobierno de los Estados Unidos a la economía norteamericana para atenuar los efectos de la crisis financiera, especialmente para el rescate de grandes empresas como la General Motors, el Citi Bank y otras.

Con la crisis financiera, el desempleo ya no coexiste con la inflación, sino con la deflación, siendo ése el escenario perfecto para la aplicación de la Teoría Keynesiana (Pavón, 2012). Pero, la deflación, es tan peligrosa e indeseable como la inflación y en ello juegan un papel muy importante las "expectativas" de los consumidores. Si las expectativas de los consumidores son hacia la baja en los precios, entonces no van a consumir en espera de que los precios bajen más. Si baja el consumo presente, los precios van a seguir bajando y posiblemente bajen más que los costos de producción de las empresas, con lo cual éstas van a perder mucho dinero y van a correr un riesgo económico extraordinario (Pavón, 2012). O sea que ni la inflación ni la deflación son deseables porque ambas son dañinas para las economías.

\subsection{La Respuesta al Desempleo en Honduras está en el Modelo Keynesiano}

Como el problema actual de la economía mundial es el desempleo y la tendencia a la deflación - y éste es el caso de Honduras - el Modelo Keynesiano calza perfecto para resolver 
esos dos problemas, por lo cual había que estimular la demanda agregada, aplicando políticas económicas expansivas, tales como bajar los impuestos, bajar los tipos de interés, incrementar el gasto público (en inversión) y devaluar, a fin de hacer las exportaciones más competitivas con el exterior, a sabiendas de que la devaluación es una solución transitoria de corto plazo, porque en el mediano plazo ésta será más perjudicial que beneficiosa, ya que el estímulo para exportar se volverá un problema para producir en el mediano plazo a través de un incremento de costos.

Para resolver ese problema, se tendría que hacer devaluaciones en cadena para mantener la competitividad de las exportaciones; pero, esto es casi una forma artificial de promoción de exportaciones, si no se corrige el problema de fondo que es el aparato productivo. El problema de fondo de la economía hondureña es el deficiente aparato productivo nacional que requiere ser transformado; es decir, se requiere de una reingeniería de la economía a fin de pasar de un modelo esencialmente "agro exportador" a uno de industrialización, en donde se produzcan productos terminados, como lo ha hecho por ejemplo Suiza (Sabillón, 2009), para luego emigrar hacia la industria pesada de tercera y cuarta generación.

Se trata, por lo tanto, de pasar de producir sólo arados o arneses a productos más sofisticados y de mayor valor agregado, tales como vehículos, computadoras, aviones, buques y potenciar la industria farmacéutica, sin descuidar la tecnología de punta y la innovación, que son fundamentales en este proceso. Esto, que parece una ilusión óptica, no lo es si los hacedores de la política económica tienen una visión de país, de mediano y largo plazo.

\section{Keynes y el Rostro Humano de la Política Económica}

Según la Teoría Keynesiana, el "punto medular" de la política económica es estimular la demanda agregada, como factor de crecimiento económico y como mecanismo para reducir el desempleo. Uno y otro objetivo tienen un contexto humano, ya que el estímulo a la demanda implica el deseo de satisfacer necesidades de la sociedad y, la reducción del desempleo, el de darle poder de compra a la población para que puedan adquirir los bienes y servicios que necesitan para su vida.

El crecimiento económico es fundamental para dar mejores condiciones de bienestar a la población, porque de esa forma, la gente tendrá trabajo y con ello ingreso, el cual es tan necesario para poder vivir y tener una vida digna. Pero, se observa una paradoja, y es que el crecimiento necesita de la ejecución de proyectos que proporcionen la materia prima a los sectores estratégicos, tales como la industria manufacturera, minería, construcción etc. dentro de los cuales está el subsector energético.

Lo paradójico es que el desarrollo del subsector energético requiere de la producción de energías renovables, y eso se puede lograr mediante la construcción de represas hidroeléctricas, porque éstas constituyen una opción viable en la producción de energía limpia, no contaminante del medio ambiente. Si se produce energía limpia mejora nuestro 
ambiente y se reversaría por completo la matriz energética de Honduras que, según el señor Presidente de la República ${ }^{7}$, está compuesta por un $60 \%$ de energía limpia y $40 \%$ de energía térmica, que es altamente contaminante del medio ambiente y mucho más cara que la energía limpia.

El grave problema de producir energía limpia, a través de proyectos hidroeléctricos, es que implica inundar vastas extensiones de tierra, mucha de la cual es tierra fértil y apta para la agricultura; con el grave inconveniente, además, que de por medio hay seres humanos que viven en esas porciones de tierra y que la ejecución de esos proyectos - que son necesarios para el desarrollo del país - los dejan sin su activo o su casa donde viven o se mata su cultura, sus costumbres y sus tradiciones a lo cual tienen derecho. Surge la duda, entonces, si el remedio no es peor que la enfermedad.

El ser humano tiene prioridad ante todo intento de desarrollo económico y el objetivo último de la economía es precisamente el bienestar del ser humano (Hermosilla Paul. G.). De aquí se concluye que sin bienestar no hay economía. Por lo tanto, si no se tiene en cuenta al ser humano, se cae en el concepto de la Economía Marginalista - especialmente el pensamiento de Léon Walras - que le dio a la economía un sentido estrictamente matemático, es decir “cabalístico" de una ciencia exacta (Anguita, 2011).

Sin embargo, la economía como ciencia social e histórica, no es una ciencia exacta sino experimental y, si los instrumentos que se utilizan para tratar de resolver los problemas económicos que afectan al ser humano y su entorno no dan los resultados esperados, pues se cambian por otros que sean más efectivos (Anguita, 2011). Según Walras, la economía tiene que crecer a toda costa porque tiene que ser rentable y, si de por medio hay seres humanos, eso no importa, la ecuación tiene que funcionar (Anguita, 2011). Pero, uno se pregunta ¿qué objetivo tiene crecer económicamente, si no es para satisfacer las necesidades del ser humano? La economía tiene un fin muy claro: el bienestar general de la sociedad, su felicidad. Annemarie Pieper señaló "la actividad económica - bien sea del individuo o del Estado - se determina por el objetivo de la maximización de los beneficios..." (De Garay, 2008).

Es relevante el hecho de que Keynes, siendo un economista de corte capitalista, en su plan de reformador de la economía y como respuesta a la Gran Depresión de los años 30’s, se centró en una receta para curar los dos problemas fundamentales de entonces, que era el desempleo y la deflación; pero, como el desempleo lleva implícito al ser humano, entonces, su modelo también puede interpretarse como la búsqueda de una solución compatible con la esencia misma del ser humano, tener un trabajo digno, ya que él entendía que el capitalismo tenía que cambiar para mejorar las condiciones de vida de la sociedad.

\footnotetext{
${ }^{7}$ Véase http://www.enee.hn/index.php/component/content/article/156-periodistas/1007-presidente-de-larepublica-seguira-de-cerca-comportamiento-del-servicio-electrico-nacional.
} 
De ahí que el fundamento de su modelo económico es la demanda agregada, definiendo ésta como la necesidad objetiva de satisfacer necesidades de la población (Anguita, 2011). Además, señaló que el pleno empleo con derechos de los trabajadores es posible; que el Estado protagoniza la macroeconomía y la iniciativa privada la microeconomía (Anguita 2011). Por consiguiente, el papel del Estado es importante porque es el que planifica y es el responsable del diseño de la política económica y, si planifica, tiene la gran oportunidad de hacer "algo" por el ser humano.

La propuesta de Keynes también es una reforma al sistema capitalista para corregir el problema de la inequidad, ya que, entre el modelo capitalista - neoliberalismo y el socialismo - populismo, se presenta también una paradoja la cual consiste en que el sistema capitalista en su etapa superior es eficiente pero muy inequitativo, lo cual propicia la pobreza y la desigualdad. En cambio, el modelo socialista, en esa misma etapa, es muy equitativo pero ineficiente.

Con el planteamiento anterior, lo fundamental es determinar qué modelo es más equitativo y eficiente que propicie una respuesta efectiva a los problemas económicos y sociales de los países, especialmente de Honduras; en otras palabras, es preguntarse ¿qué opción le queda a la sociedad y a las futuras generaciones? Y la respuesta quizá sea la adopción de un modelo de "Economía Social de Mercado" (economía mixta) el cual ha sido practicado por los países nórdicos y Alemania, con excelentes resultados hasta la fecha. En la actualidad se observa cómo países comunistas - como China Continental y Cuba - están modificando la estructura del sistema comunista, introduciendo un estilo económico capitalista dentro del mismo sistema comunista. Esto sencillamente demuestra una realidad "nada es eterno en la vida, todo es cambiante" y por eso la innovación toma mayor sentido, ya que ambos sistemas (capitalista y comunista/socialista) requieren de ajustes en sus estructuras; requieren ser renovados e innovados.

Otra de las grandes preocupaciones de Keynes fue una mejor distribución del ingreso con fiscalidad, puesto que él pensaba que el sistema tributario tenía que ser progresivo, con el cual los ricos pagarían más y los pobres menos (Modelo de Robín Hood) para favorecer el gasto público en inversión en favor de las mayorías. Sin embargo, lo que se observa actualmente es todo lo contrario, ya que en casi todos los países de América Latina se tienen modelos con fiscalidad regresiva; aunque, sí progresivos hacia mayores impuestos a la población, especialmente impuestos indirectos, ya que los estados necesitan de mayor captación de ingresos - especialmente los centroamericanos - que, tal como lo ha señalado el economista Luis René Cáceres, los sistemas tributarios regresivos agravan el problema de la desigualdad en la distribución del ingreso y de la riqueza (Cáceres, 2013).

El otro aspecto humano de Keynes es la atención al triple componente del salario global, que puede ser directo, indirecto y diferido. El empleo directo está compuesto por los sueldos y salarios devengados por los trabajadores; el indirecto por la educación y la salud, a lo cual 
habría que agregar una excelente infraestructura pública y servicios públicos de alta calidad; y, el salario diferido, por las pensiones y jubilaciones (Anguita, 2011). Sin embargo, lo que se aprecia en casi toda Latinoamérica es que ese triple componente del salario global, en vez de mejorar, va en franco deterioro

Lamentablemente todas estas brillantes ideas de este connotado economista capitalista fueron "desconocidas" por los liberales y neoliberales, ya que hoy de lo que se trata es de reducir lo más posible el salario directo e indirecto - contrario a las enseñanzas Keynesianas - y en debilitar el sistema de pensiones y jubilaciones, una especie en peligro de extinción en América Latina. De aquí nace la idea - y que hay que entender ahora - que los sistemas de pensiones en casi todos los países de la región están a punto de colapsar iiQué mal estamos! El ser humano ha pasado a un segundo plano; mientras, lo importante es la rentabilidad de la actividad económica y la pregunta es ¿quién se beneficia de esa rentabilidad?

La práctica del Modelo Keynesiano, desde mediados de los años 30’s hasta 1973, dio la oportunidad a los países de la región de aprovechar la fuerte onda expansiva generada con la nueva economía (Anguita, 2011); pero, los países de la región, por los motivos que sean, no supieron aprovecharla para crear más fuentes de trabajo ${ }^{8}$.

Lo mismo aplica para la década de los 90`s, que fue la época de la postguerra en la región centroamericana, dentro del auge del Consenso de Washington, que trajo consigo el desbaratamiento de las barreras económicas, entre las cuales está el libre comercio como vehículo de desarrollo para los pueblos. Pero, como muy bien señala Ha- Joon Chang, la práctica del Modelo Neoliberal estableció "el libre comercio" como base del desarrollo y eso requiere de la reducción de los aranceles para aprovechar las ventajas del mercado. Sin embargo, esto ha perjudicado mucho a los países menos desarrollados - entre ellos los centroamericanos - por la pérdida de ingresos presupuestarios.

Así mismo, señala que "los países que hoy son desarrollados (PHD), como Alemania, Austria, Bélgica, Dinamarca, España, EE. UU., Francia, Holanda, Italia, el Reino Unido, Suecia y Suiza, en su proceso de crecimiento acelerado usaron políticas comerciales e industriales intervencionistas para promover sus industrias nacientes y alcanzar las economías de primera línea" (Chang, 2003) y eso contribuyó, en gran medida, en su proceso de desarrollo. Por eso, en su artículo, que es una crítica al consenso de Washington y, consecuentemente, al modelo neoliberal, concluye:

"Esto significa que los países en desarrollo de hoy necesitan aranceles mucho más altos que los usados por los PHD en épocas anteriores, si quieren proporcionar un grado de protección real a sus industrias, similar al que tuvieron las industrias de los PHD en el pasado" (Chang, 2003).

${ }^{8}$ Anguita trata este mismo concepto para el caso de España 
En cuanto a las inequidades existentes, Keynes entendía que en el mundo hay grandes desigualdades en los ingresos y en la riqueza, al grado de señalar que "por mi parte creo que hay justificación social y psicológica de grandes desigualdades en los ingresos y en la riqueza”, pero también afirmaba que no son justificables “...tan grandes disparidades como existen en la actualidad" (Gaviria Ríos, 2007). Por eso Keynes enfatizó que los dos graves problemas del capitalismo son el desempleo involuntario y la generación de una distribución tan inequitativa de la riqueza y el ingreso (Gaviria Ríos, 2007).

Por su parte, Angus Deaton (2013) en su obra "El Gran Escape" afirma contundentemente que "hoy en día el mundo es crecientemente inequitativo" e igualmente señala la existencia de "una paradoja: los mecanismos que durante los dos últimos siglos y medio permitieron a los países industrializados experimentar un progreso sostenido, son los mismos que ha ensanchado la brecha entre los que salieron avante y los que se vienen quedando atrás". Así, él llega a la conclusión de que "el desarrollo económico es a su vez causa y efecto de la gran desigualdad social de nuestros días” (Deaton, 2013).

Thomas Piketty, por su parte, reúne y analiza la distribución del ingreso y de la riqueza en una veintena de países desde finales del siglo 18 hasta nuestros días y su conclusión es que "la concentración extrema de los patrimonios amenaza los valores de la meritocracia y de la justicia social de las sociedades democráticas" (Piketty, 2015). Además, agrega "Las desigualdades siempre han sido una fuente de preocupación, pero lo nuevo en este libro es que reuní una gran cantidad de datos históricos. Hasta hace poco, había relativamente pocas pruebas" (Piketty 2015).

Lo novedoso es que Piketty, en su obra, aboga por un sistema tributario progresivo, aumentando fuertemente la carga tributaria a los ricos porque argumenta que "cada nación ha respondido de manera diferente a una ley básica del capitalismo, según la cual el rendimiento del capital suele ser superior, a veces por mucho, a la tasa de crecimiento de la economía, lo que puede estimular la concentración de la riqueza y agravar la inequidad" (Piketty, 2015).

Honduras enfrenta las mismas preocupaciones, ya que las desigualdades en la distribución del ingreso y de la riqueza han empeorado en los últimos tiempos, especialmente después de 1990, cuando se inicia la práctica del Modelo Neoliberal. Para mitigar este problema, es necesario que el Gobierno de Honduras implemente un "Pacto Fiscal", que dé paso a una "Reforma Tributaria" con carácter progresivo y se combata la corrupción, para que con los recursos tributarios captados, se le retribuya a la sociedad hondureña la deuda social, financiando y ejecutando proyectos de alto contenido social (Por ejemplo, buenas carreteras, servicios públicos de calidad, excelente sistema de servicio de transporte público, subsidios para estudiantes de escasos recursos, buen sistema de seguridad social, buen sistema educativo, entre otros) que beneficien a un mayor número de personas, que les permita gozar de mayores niveles de bienestar . 
La imposibilidad de que el Modelo Keynesiano diera una respuesta a la crisis petrolera de 1973 (Keynes no la contempló), hace aparecer el Modelo Neoliberal de Milton Friedman, el cual es adoptado por casi todos los países y Honduras no fue la excepción, ya que a través del "Consenso de Washington" - implementado por el Fondo Monetario Internacional Honduras lo adopta a partir de 1990.

Estudios efectuados para medir el impacto de este modelo en Honduras, dan cuenta que, si bien ha tenido algunos beneficios, las desventajas superan con creces a aquellos, puesto que ha contribuido a una mayor concentración del ingreso y de la riqueza, generado mayor desempleo y pobreza y, consecuentemente, a que un buen sector de la población no tenga ingresos monetarios para suplir sus necesidades básicas, favoreciendo la delincuencia, el crimen y la inseguridad, con un enorme costo social para el país.

Lo paradójico es que, si parte del dinero que se ha gastado en seguridad y combate a la violencia, se hubiese invertido en educación y salud en los últimos 6 años (que forma parte del salario indirecto de los trabajadores), seguramente que los frutos que se tendrían en cuanto a indicadores sociales (educación, salud, reducción de la pobreza) serían mejores, con menor desempleo y mayor bienestar para la población.

Según cifras plasmadas en el Presupuesto Ciudadano para 2016, la partida presupuestaria para seguridad y defensa alcanza un monto de L.13,340.5 millones (SEFIN, 2016); y las pérdidas causadas a las empresas por robo y actos delictivos, según la Federación Centroamericana de Transporte de Carga (Fecatrans), alcanza un monto equivalente a US\$150.0 millones anuales, es decir L.3,390.0 millones de lempiras al año (Diario La Prensa, 2014).

Colocando todo junto, se habla de L.17,000.0 millones anuales, una cifra que representa el $13.6 \%$ del ahorro nacional, considerando éste como el M3, una definición ortodoxa de dinero que incluye: el medio circulante (billetes y monedas en poder del público) más los depósitos en cuentas de cheques, más los depósitos en cuentas de ahorro, más los depósitos en cuentas a plazo fijo. Obviamente, una definición más estricta de dinero, debería incluir los depósitos en moneda extranjera en el sistema bancario nacional más otros certificados de tenencia de dinero, como serían los bonos del Estado. Si se relaciona ese gasto con el PIB ello representa el $3.7 \%$.

\section{Las Limitantes del Modelo Keynesiano para Resolver el Alto Desempleo en Honduras.}

Dadas las características recesivas de la economía mundial, manifestadas a través de un bajo y lento crecimiento económico, por deficiencias de demanda agregada con tendencia a la 
deflación (con excepción de algunos países) debido a la caída en el precio de petróleo a niveles de $\$ 45-\$ 50^{9}$ por barril, se puede concluir también que este es el caso de Honduras.

La economía hondureña muestra un lento y bajo crecimiento del $3.6 \%$ anual, al 31 de diciembre de 2015, que al compararlo con la tasa de crecimiento poblacional del $2.0 \%$ anual (según el último censo de población del INE) prácticamente deja nada para el desarrollo. En concreto, lo que se tiene en Honduras es un lento crecimiento que impacta fuertemente en un aumento de la tasa de desempleo, acompañado de una tendencia clara a la deflación, al situarse la tasa de inflación a diciembre de 2015 en 2.4\% (Véase Figura 3). La causa de estos dos fenómenos es la deficiencia en la demanda agregada.

Bajo este panorama, la política keynesiana sí tiene vigencia para resolver los problemas del alto desempleo en el país, a través del estímulo a la demanda agregada. La Figura 2 siguiente, muestra el crecimiento del PIB real de Honduras para la serie 2005 - 2015.

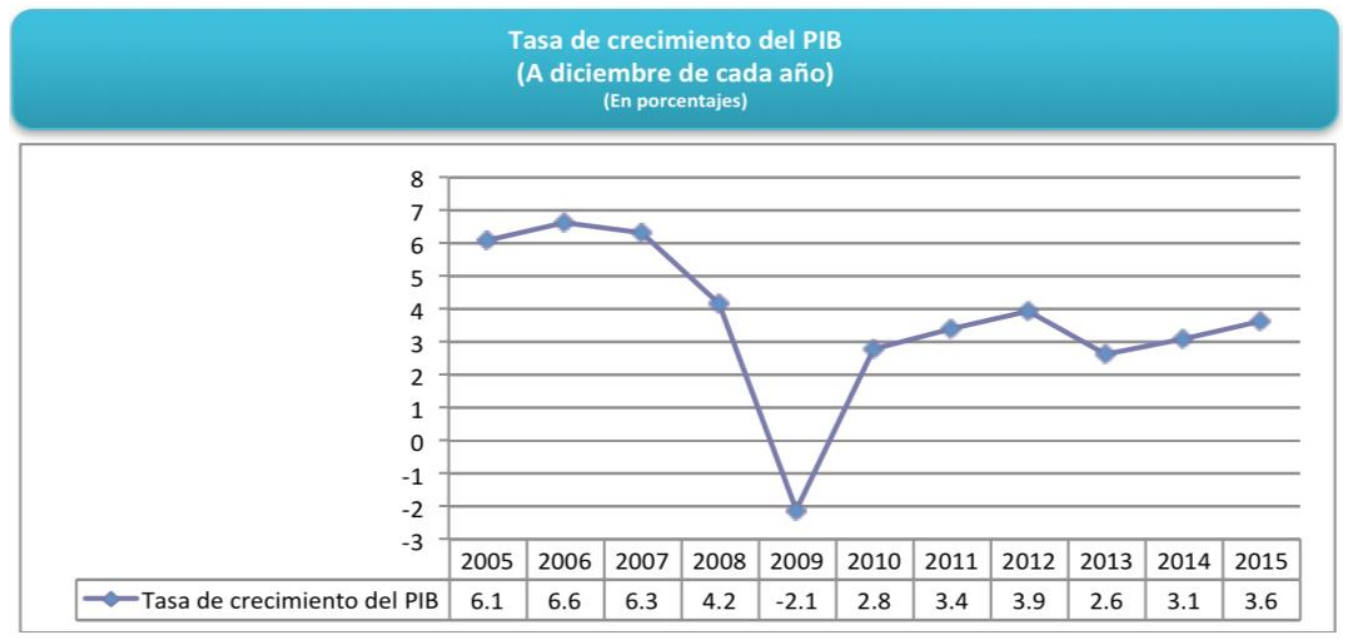

Figura 2. Tasa de Crecimiento del PIB

Fuente: Elaboración propia con datos del BCH.

Al comparar los nueve años de la serie, que se presentan en la Figura 3, la economía hondureña refleja una baja tasa de inflación con tendencia a la deflación, lo cual se explica por la caída en el ingreso familiar originado por la falta de empleo, que reduce la demanda agregada; aunque hay que tener en cuenta que, en alguna medida, este efecto está siendo compensado por el ingreso de remesas familiares, que según estimaciones del $\mathrm{BCH}$, en el 2016 serán cercanas a los US $\$ 4,000.0$ millones.

\footnotetext{
9. Según el Portal Precio del Petróleo en: http://www.preciopetroleo.net/ el WTI toca los 48,43 dólares y el
} Brent los 49,85 dólares 
Sin embargo, y tal como lo ha señalado Luis R. Cáceres (Cáceres, 2013), las remesas familiares constituyen una trampa, porque el auge de éstas podría revertirse en el corto y mediano plazo, si se diera en los Estados Unidos el fenómeno de una recesión con deflación, que no es una lejana utopía o, si el presidente electo de los Estados Unidos, el Señor Donald Trump, cumpliera sus promesas de campaña de endurecer la política migratoria y se diera una serie de masivas deportaciones de indocumentados. La Figura 3 siguiente, muestra la tendencia de la inflación anual desde diciembre 2006 a diciembre 2015.

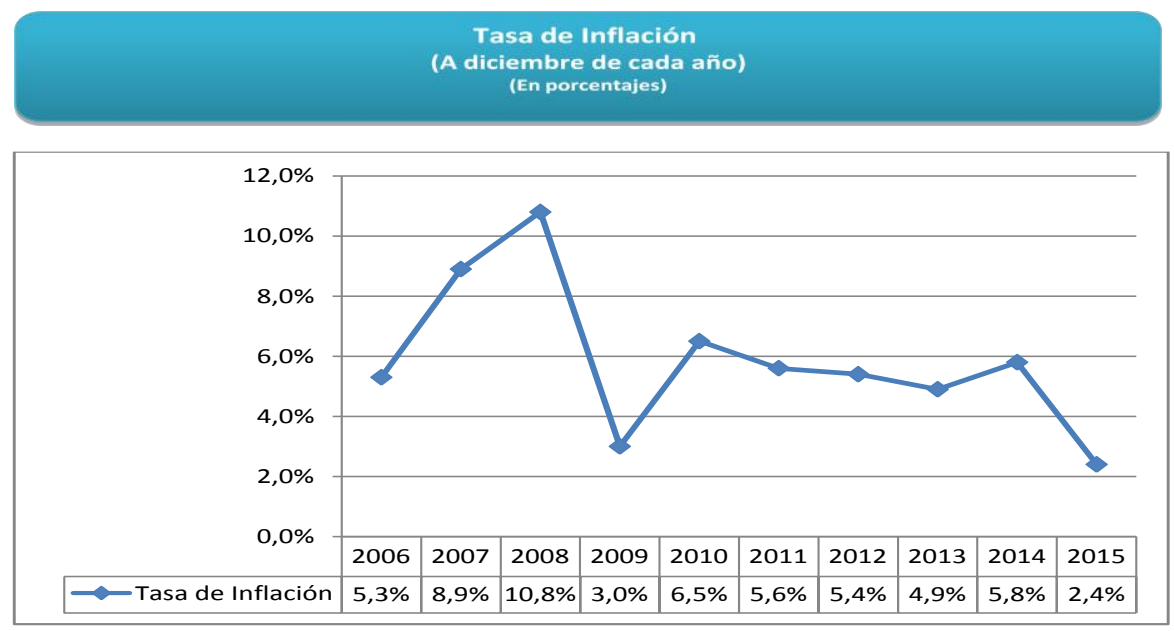

Figura 3. Tasa de Inflación

Fuente. Elaboración propia con datos del BCH.

Teóricamente, el Modelo Keynesiano resolvería la situación del alto desempleo en la economía hondureña y la tendencia a la deflación; pero, mi investigación me ha llevado a concluir, que este modelo es difícil de aplicar en el corto plazo, debido a que la economía hondureña presenta ciertas condicionantes que limitan, por el momento, el margen de acción de este modelo. ${ }^{10}$

${ }^{10}$ De acuerdo a datos proporcionados por el Lic. Edwin Araque Bonilla, Expresidente del BCH, hay un antecedente bastante reciente de uso de políticas keynesianas que vale la pena destacar. En el periodo del 2006 al 2008 se implementó una política cuasi-keynesiana con buenos resultados en materia de crecimiento, aplicando una política monetaria expansiva, mediante la cual las operaciones de mercado abierto del $\mathrm{BCH}$ (ventas de bonos) se redujeron de L 25,000 millones a L 8,000 millones, aproximadamente; la tasa de política monetaria se redujo de $12 \%$ a $3.50 \%$, lo que incidió en una baja en la tasa de interés de los bonos y letras del sector público de $12 \%$ a 5\%-6\% en promedio; esto implicó una caída considerable en las tasas de interés en el sistema financiero.

Con este efecto de transmisión de la política monetaria del $\mathrm{BCH}$, se estimuló la inversión en todos los sectores productivos hasta obtener un crecimiento histórico del 6.6\% (PIB) no registrado en la historia económica reciente de Honduras. Asimismo, se creó un fideicomiso de más \$500 millones administrado por BANHPROVI con recursos del $\mathrm{BCH}$, y se inyectaron recursos a la economía por más de \$220 millones con recursos de Petrocaribe y ALBA. Por el lado de la política fiscal, se aumentó la inversión pública por más de L 15,000 millones y no se aplicó impuesto nuevo alguno durante este periodo, a pesar de que el 2008 aumentó del precio del petróleo en el mercado internacional que alcanzó un precio de \$148 el barril. Aún con todo, se otorgaron 


\subsection{Condicionantes de la Economía Hondureña}

Los excesos en el gasto público corriente y la corrupción en los últimos 6 años (efecto rezago), hacen que ahora se deben tomar medidas contractivas para eliminar los desequilibrios causados en la economía por dicho efecto, como es el alto déficit fiscal del 7.9\% en 2013; el alto déficit comercial, que se ha casi triplicado en los últimos 5 años; y el déficit de la cuenta corriente de la balanza de pagos, que se ha duplicado, han originado un efecto de contrapartida en la balanza de pagos, que implica pérdida de Reservas Internacionales por parte del $\mathrm{BCH}^{11}$. Es importante observar que la brecha comercial en los últimos años se va ampliando y que la pendiente de la curva de las importaciones es más inclinada que la de las exportaciones, lo cual refleja una mayor propensión marginal a importar por parte de la economía hondureña, lo que justifica una vez más, una modificación a la estructura productiva del país con la celeridad del caso, ya que el problema reside en ésta. Recalcando, el problema es la deficiente estructura productiva del país que es la que hay que reinventar.

En las Figuras 4 y 5 siguientes, se presenta el deterioro de algunos indicadores claves, como el déficit fiscal, la balanza comercial y la brecha comercial, durante los últimos 9 y 11 años, respectivamente:

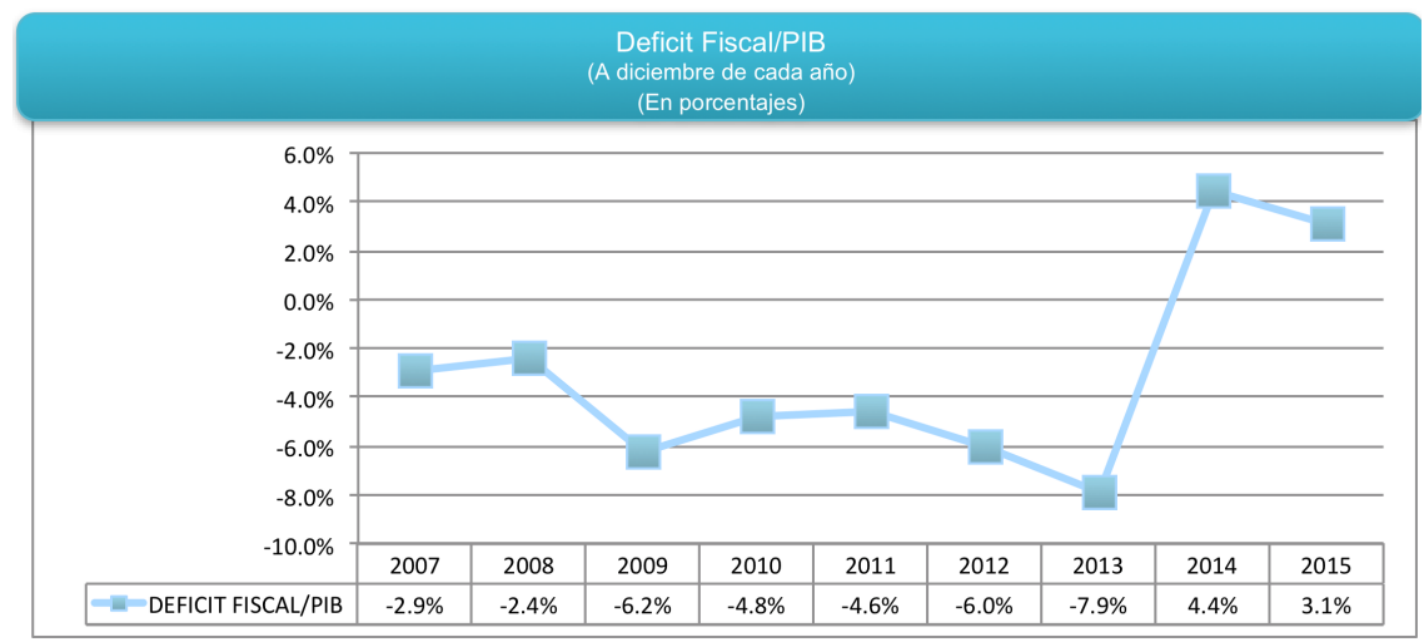

Figura 4. Déficit Fiscal/PIB

Fuente: Elaboración propia con datos se SEFIN.

subsidios a los precios de los combustibles para amortiguar el impacto inflacionario y el tipo de cambio se mantuvo fijo en L.18.8951 con 0\% de riesgo cambiario.

${ }^{11}$ En la actualidad las cifras han mejorado, ya que los saldos de las Reservas Internacionales Brutas al 28 de junio de 2016 eran de US\$4,202.6 millones y las Reservas Internacionales Netas de US\$3,906.9 millones. La mejora en la Reserva Internacionales a junio, comparadas con las de diciembre de 2015, se atribuye al aumento del precio del café y en el ingreso de remesas familiares, la caída del precio del petróleo y, fundamentalmente, a la caída de la demanda agregada de los principales sectores productivos. 


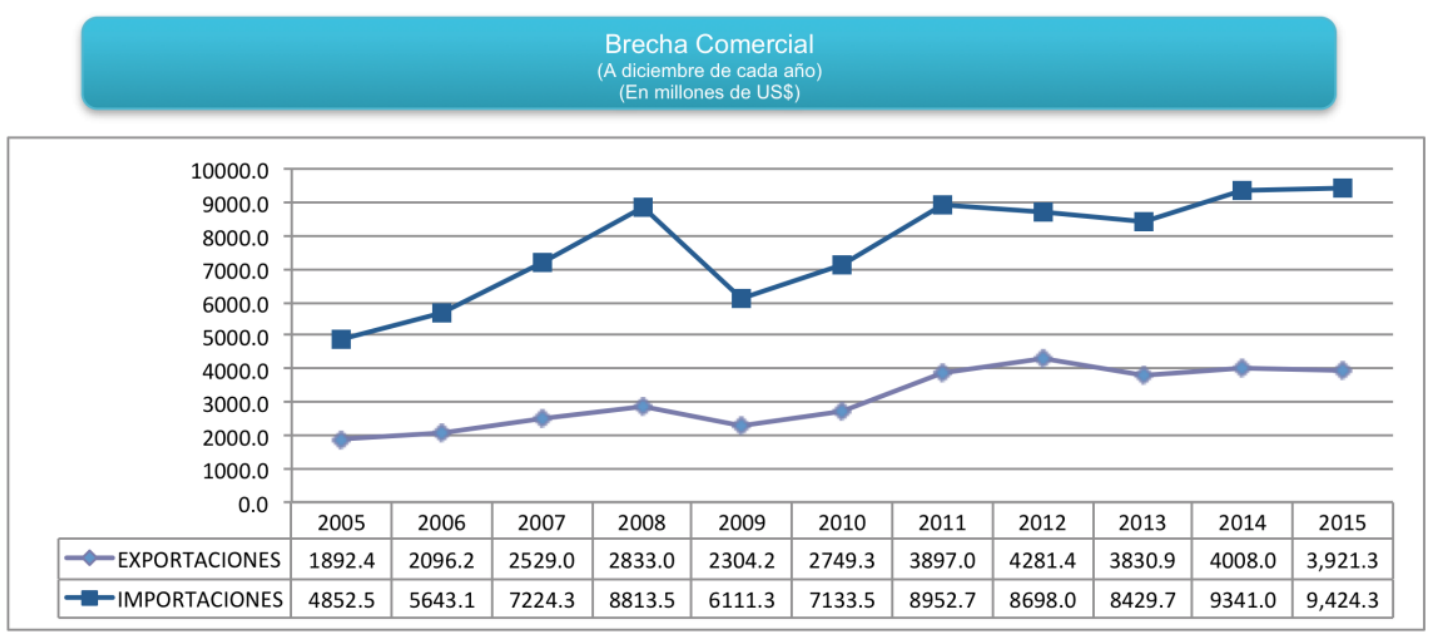

Figura 5. Brecha Comercial

Fuente: Elaboración propia con datos del Banco Central de Honduras

Honduras ha decidido continuar con la práctica del Modelo Neoliberal que privilegia las políticas de oferta agregada y no de demanda agregada. Sin embargo, el señor Adolfo Facussé ha señalado que en Honduras lo que se tiene es una mezcla de cosas debido a la alta intervención del gobierno en la economía y al exagerado gasto público corriente ${ }^{12}$, por lo que según su análisis, el país ya no se rige por el Modelo Neoliberal (Facussé, 2013).

Pero, aún y cuando el señor Facussé tuviese la razón, la evidencia empírica señala que el tipo de políticas que se está utilizando son de corte neoliberal porque son de estímulo a la oferta agregada y, por ello, la política económica está orientada a medidas contractivas y no expansivas, que no favorecen la reducción del alto nivel de desempleo, porque se orientan a reducción de costos de producción de las empresas, como la reducción en la contratación de mano de obra y estancamiento de los salarios.

Por eso, se recortan o se congelan los salarios, se cierran empresas, se baja el gasto público en inversión, se suben las tasas de interés, se aumentan o se crean nuevos impuestos, todo lo cual incide en una reducción del poder de compra de los ciudadanos que provoca un menor consumo y una menor inversión, lo cual, aunado a un menor gasto público en inversión y a un deslizamiento limitado del tipo de cambio, origina una mayor tasa de desempleo.

La economía hondureña presenta características "endógenas" propias que limitan el accionar de la política económica. Dentro de este concepto se tiene que es una economía pequeña, abierta y con un sistema de tipos de cambio fijo, que fluctúa dentro de una banda de flotación o Sistema de "Crawling Peg", lo cual restringe la acción de la política económica en el logro

\footnotetext{
${ }^{12}$ Véase ponencia del Señor Facussé titulada "El Modelo Económico Neoliberal y un Modelo Alternativo para Honduras; así como "El Neoliberalismo en Honduras. Lecciones de más de dos décadas.
} 
de los principales objetivos económicos del gobierno, porque el $\mathrm{BCH}$ tiene claramente un objetivo cambiario.

Las características de "endogeneidad" de la economía hondureña, se explican por lo siguiente: a) Por economía pequeña se entiende no tener poder de influencia en los precios internacionales y, por tanto, Honduras como país es tomador de precios que se fijan en el exterior para sus bienes transables internacionalmente; b) Por economía abierta se entiende que tiene relaciones económicas con el resto del mundo, lo cual afecta la balanza comercial y la balanza de pagos.

En el caso concreto de la balanza comercial, el déficit comercial aumenta, porque las importaciones son mayores que las exportaciones. El desequilibrio comercial conduce a que el $\mathrm{BCH}$ pierda reservas internacionales $\mathrm{y}$, al perder reservas, se incrementa el riesgo cambiario; c) Por tipo de cambio fijo se entiende que Honduras tiene un sistema de tipos de cambiario fijo, que fluctúa dentro de una "Banda de Flotación", por lo cual el Banco Central está obligado a mantener Reservas Internacionales Netas (RIN) equivalentes a por lo menos tres meses de importaciones (Valor de referencia del FMI); es decir, que la política monetaria hondureña tiene un claro objetivo cambiario, lo cual limita el margen de acción. Además, utilizando la política cambiaria, hay un objetivo de control inflacionario, el cual se opera a través de la subasta pública de divisas, como "ancla" nominal para atenuar la demanda agregada.

Como el país decidió pedir la asistencia del Fondo Monetario Internacional, entonces debe cumplir con el acuerdo firmado con ese organismo. Ese acuerdo somete a la economía hondureña a adoptar medidas de política económica restrictivas y no expansivas ${ }^{13}$, que es lo contrario a lo recomendado por Keynes para corregir la situación de desempleo. Por tanto, no es de extrañar, que, en lugar de reducir el alto desempleo en el país, éste aumente cada vez más.

Las medidas que el Gobierno de Honduras está tomando son medidas antiinflacionarias en un momento en que lo que el país presenta es un proceso deflacionario. La lucha contra la inflación, según Keynes, exige medidas presupuestarias contractivas, tales como incrementos de impuestos, incrementos de tasas de interés y reducción del gasto público, porque se trata de disciplinar el gasto público. Por tanto, bajo estas circunstancias, no debería haber déficit fiscal, ni deuda pública. Pero, en Honduras, si bien el déficit fiscal se ha reducido, aún está por encima de la estándar internacional de sostenibilidad del déficit; y la situación de la deuda pública (externa e interna) se ha incrementado, ya que según datos de SEFIN la relación

\footnotetext{
${ }^{13}$ Según información del Lic. Araque, hay que hacer la salvedad de que en el periodo 2006-2009, se rompió el acuerdo con el FMI porque en la gestión del Ex presidente Zelaya se aplicó una política monetaria y fiscal expansiva (neo keynesiana) lo que trajo como consecuencia el incumplimiento del acuerdo con el FMI, que presionaba por la devaluación y la contracción de la economía mediante políticas monetaria y fiscal contractivas.
} 
deuda pública total a PIB a diciembre de 2015 y primer trimestre de 2016 fue de $46.1 \%{ }^{14}$ (Diciembre 2014 era de 45.6\%).

Como la economía hondureña tiende a la deflación con un alto nivel de desempleo, mi investigación me ha lleva a concluir que las medidas que se están tomando son contrarias a la solución del problema de la deficiente demanda agregada y, por el contrario, provocan un mayor desempleo.

Si Honduras no tuviera el problema de los graves desequilibrios macroeconómicos estructurales, agravados por el excesivo gasto público en el último sexenio (efecto rezago), que son los que hoy se están tratando de corregir con la asistencia del Fondo Monetario Internacional (FMI), la política económica podría utilizar la baja de impuestos y el incremento del gasto público en inversión para corregir el desempleo y podría aumentar el déficit fiscal y la deuda pública casi indefinidamente para resolverlo. Pero, actualmente, hacer eso no es factible.

Es un hecho que, con las medidas que se están adoptando, el efecto es de más desempleo; aunque se estima que bien se puede re-direccionar la política económica hacia mayores logros con voluntad política, a fin de no privilegiar tanto el crédito al consumo y comercio que, junto al sector de energía y telecomunicaciones, absorben más del $75 \%$ de la cartera de crédito de los bancos. Esta situación beneficia más al sistema bancario nacional, en detrimento de otros sectores de población que no tienen acceso al crédito, ya que la incidencia de esos sectores sobre el valor agregado es mínima, en relación a las actividades productivas de la micro y pequeña empresa y al sector agropecuario, que tienen un mayor impacto en la absorción de mano de obra, porque son más intensivos en este factor de producción.

Así mismo, el crédito al sector de energía y telecomunicaciones es corporativo y los montos de crédito son considerablemente altos, por lo que cuatro o cinco préstamos pueden absorber más de 5,000 millones de lempiras, lo que en la micro y pequeña empresa podría significar el otorgamiento de entre 500 y 1000 préstamos, con montos de alrededor de los 500,000 lempiras en cada caso.

Por otra parte, el crédito al comercio y al consumo es nocivo, porque reduce las reservas monetarias internacionales y presiona a la devaluación y se vuelve un círculo vicioso de inflación-devaluación-inflación. Finalmente, este tipo de crédito hace a la economía hondureña más dependiente del exterior porque la balanza de pagos es abierta por prescripción del FMI.

Lamentablemente, ésta es la realidad del país en este momento. Con un desempleo total a diciembre del 2015 del $62.4 \%{ }^{15}$ (IIES, OEE, UNAH, 2016) Honduras necesitaría políticas

\footnotetext{
${ }^{14}$ Dato tomado de SEFIN, del Cuadro "Indicadores de riesgo del Gobierno Central" 2016

15 Según el Observatorio Económico y de Emprendimiento-OEE-IIES de la UNAH, el desempleo real para el 2016 se compone de la tasa de desempleo abierto de $7.3 \%$, la tasa de desempleo invisible de $41.1 \%$ y la tasa de subempleo visible del 14.1\%. Haciendo una comparación con el 2014, la situación del desempleo se ha deteriorado, ya que en ese año la situación era para la tasa de desempleo abierto $5.3 \%$, para la tasa de desempleo invisible el $27.7 \%$, y para la tasa de subempleo visible el $12.5 \%$.
} 
expansivas para estimular la economía; pero, el acuerdo con el FMI y las características propias de "endogeneidad" de la economía hondureña, no le permiten adoptar ese tipo de medidas, porque hay que volver a la disciplina económica y estabilizar la economía, que implica que el déficit fiscal no debe superar el 2\% del PIB y la deuda pública total con respecto al PIB no debe superar el 50\%, que es el Parámetro de Sostenibilidad de la Deuda.

Si bien es cierto que el déficit fiscal se ha ido reduciendo, al pasar de $-7.9 \%$ en el 2013 a $3.1 \%$ en el 2015 (Ver Figura 4), éste debe seguir bajando hasta alcanzar el punto de sostenibilidad del $-2 \%$ y mantenerlo en ese porcentaje por un largo periodo de tiempo. Entonces, la situación en que se encuentra la economía hondureña actualmente es que, para reducir el alto nivel desempleo, habría que aplicar medidas de carácter expansivo, de acuerdo a la tesis keynesiana; pero, el excesivo gasto público corriente del último sexenio, que hizo que el déficit fiscal se incrementara hasta el $-7.9 \%$ en 2013, implica que ahora lo que se necesita es disciplina fiscal. De ahí que lo que hay que hacer, de acuerdo al FMI, es subir impuestos, subir tasas de interés y reducir el gasto público, con lo cual lo que se está generando, como ya se mencionó, es más desempleo.

Este es el dilema fundamental en el que se encuentra sumida la economía hondureña en la actualidad, el cual es de difícil solución, porque con la política económica keynesiana se tendría que tener políticas expansivas para corregir el desempleo; pero, de acuerdo a la situación del país, por el excesivo gasto corriente del sector público y la corrupción, se tienen que adoptar políticas contractivas para estabilizar la economía, agravando el problema del desempleo.

\section{Conclusiones y Recomendaciones}

A través de mi investigación he llegado a la conclusión de que, en el corto plazo, no se puede reducir el desempleo en Honduras mediante el uso de una política monetaria y fiscal expansivas (estilo keynesiano) porque es necesario, primero, estabilizar la economía reduciendo los graves desequilibrios macroeconómicos estructurales, los cuales no permiten la generación de ahorro para la inversión y la generación del empleo productivo. Es por eso que el Gobierno de la República pidió la asistencia del FMI.

La eliminación de tales desequilibrios exige medidas de política monetaria y fiscal contractivas, aunque hay que tener en cuenta que el uso de la política monetaria sólo puede influir en las variables reales, como la producción y el empleo en el corto plazo; ya que en el largo plazo, las variables monetarias no pueden afectar variables reales en forma permanente, por lo que la respuesta al alto desempleo habría que buscarla en su propio mercado, es decir en el mercado de trabajo ${ }^{16}$.

\footnotetext{
${ }^{16}$ Según Cavazos Lerma, hay que actuar sobre la oferta y demanda de mano de obra, a través de mejoras en la productividad del trabajador, que incluye entrenamiento, programas de adiestramiento en el trabajo, mejoras en la educación formal, mejoras en la información del mercado de trabajo, facilitar la migración hacia aquellas zonas donde se requiere más mano de obra, eliminación de prácticas monopólicas o monopsónicas que
} 
Por de pronto, el país debe continuar con las medidas de estabilización propuestas por el FMI y, después de que la economía se haya estabilizado, empezar a soltar paulatinamente una política económica expansiva, bajando los impuestos directos, reduciendo las tasas de interés en la medida de lo posible para fortalecer la inversión privada, y subir el gasto público en inversión; al tiempo de acelerar un poco el deslizamiento de la moneda, a fin de hacer la economía más competitiva ${ }^{17}$, aunque esto sólo es recomendado por un período de tiempo limitado, ya que de nada serviría estar devaluando el lempira, si no se corrige el problema principal, que es el desarrollo y reingeniería del aparato productivo hondureño.

Dado que una política económica expansiva puede conducir a altos déficit fiscales y a un aumento de la deuda pública (Pavón, 2012) es importante adoptar una resolución constitucional, como lo tiene el Estado de Chile, para blindar el despilfarro de recursos, estableciendo "topes" a estos indicadores, tales como mantener, en todo momento, un déficit fiscal no mayor del 2\%; y una deuda pública dentro de los límites de sostenibilidad de deuda, cuyo parámetro para la deuda pública total en relación al PIB es del 50\% como máximo y, para la deuda externa en relación al PIB el $40 \%$ como valor máximo.

La otra parte fundamental de la ecuación del crecimiento es promover la oferta agregada mediante el estímulo productivo, no sólo del sector agropecuario, sino también mediante una "reingeniería" económica adoptando políticas públicas de estímulo al sector industrial, con el objeto de producir bienes terminados, en donde Honduras podría tener un salto cualitativo y cuantitativo en su desarrollo. También es fundamental una visión política de crecimiento y desarrollo de mediano y largo plazo, mediante la cual se permita concebir una plataforma de desarrollo a gran escala en el menor plazo posible, al estilo de países que han tenido un desarrollo impresionante como Qatar, Botsuana y Singapur (Sabillón, 2009)

Hay una verdad que es válida en cualquier país del mundo y es que, con una buena disciplina financiera del Estado, se puede convertir un desierto en un paraíso (Sabillón 2009) y eso es verdad en Honduras, en China, en Japón. Y, algo más importante aún, es que esta afirmación no sólo es válida a nivel macroeconómico, sino también a nivel empresarial e individual. Pero, para lograr una buena disciplina financiera del Estado de Honduras es necesario fortalecer la institucionalidad, la aplicación de la ley, la transparencia de la gestión pública,

encarecen artificialmente la mano de obra; así como también cambios de mentalidad. Sobre esta última es muy importante cambiar la cultura de nuestra gente para pasar de un concepto de esperar a "que todo me lo den" a una cultura de emprendedurismo y generadores de empleo. Es, en otras palabras, enseñarle a la gente a pescar, en vez de darle el pescado.

${ }^{17}$ Según las cifras del BCH, en la actualidad el Índice del Tipo de Cambio Real (ITCER), registra una ganancia teórica de la competitividad; es decir que la tasa de cambio del lempira con respecto al dólar está subvalorada. El porcentaje de devaluación anual en los años posteriores a la adopción de la Banda Cambiara (25 de julio de 2011) ha oscilado entre $4 \%-5 \%$. 
eliminar la corrupción y fortalecer el sistema democrática con reformas institucionales y administrados por funcionarios probos y respaldado por la MACIH y la sociedad civil.

Concluyo este artículo con un mensaje de Robert Kiyosaki, el cual es una verdad absoluta, válida, no sólo para las personas naturales y jurídicas, sino también para los gobiernos de los Estados, al afirmar lo siguiente: "Al final, ser rico, de clase media o pobre depende mucho de nuestra propia decisión. Cada día nosotros elegimos qué hacer con nuestro dinero. Con cada dólar que llega a su bolsillo, usted y sólo usted, decide qué hacer con él. Derróchelo y decidirá ser pobre, adquiera pasivos pensando que son activos y decidirá ser de clase media; inviértalo o ahórrelo y estará construyendo sus activos y su riqueza” (Kiyosaki, 2008).

\section{Bibliografía}

Aranguren Javier y Ricardo Yepes Stork (1999). Fundamentos de Antropología (4a . Edición), Editorial S.A. EUNSA, Ediciones Universitarias de Navarra.

Araque Bonilla Edwin (2016). Texto de revisión, información adicional y comentarios al borrador de este artículo. Profesor de Postgrado de UNITEC, Campus Tegucigalpa.

Anguita Julio (2011). Crisis Financiera y Desorden Mundial, dentro del Programa II Jornadas Universidad Compromiso Social y Mundo actual. Madrid, España. https://www.youtube.com/watch?v=1QTnnu9G8Cs

Banco Central de Honduras, Página WEB www.bch.hn, Principales Indicadores Económicos e ITCER. Serie de años 2005 - 2015.

Banco Central de Honduras, Página WEB www.bch.hn, Informes y Publicaciones

Cavazos Lerma (1979). Inflación, Recesión y Política. Apuntes sin editar usados en el Curso de Políticas y Programación Financiera, Centro de Estudios Monetarios Latinoamericanos (CEMLA). México, D.F.

Cavazos Lerma Manuel (1979). Teoría y Política Monetaria. Apuntes sin editar usados en el Curso de Políticas y Programación Financiera, Centro de Estudios Monetarios Latinoamericanos (CEMLA). México D.F.

Cáceres Luis René (2013). Las Tendencias al Estancamiento de las Economías Centroamericanas.

Diario La Prensa (2014), Honduras, 28 Octubre.

Deaton Angus (2015). El Gran Escape. Salud, riqueza y los orígenes de la desigualdad. Traducción de Ignacio Perrotini - México, FCE 
De Garay Jesús (2008). Filosofía del Mercado: El mercado como forma de comunicación. Fundación Caja Sur. Plaza y Valdés Editores.

Facussé Adolfo J. (2013). Presentación sobre El Modelo Económico Neoliberal y un Modelo Alternativo para Honduras. Asociación Nacional de Industriales de Honduras. Panelista, UNAH.

Giorgio Valentini (2016). Representante del Banco Mundial en Honduras. Informe Desatando el potencial económico para mayores oportunidades. Publicado en laprensa.hn, Tegucigalpa, Honduras.

Gaviria Ríos, M.A. (2007). Apuntes de teoría y política monetaria. Edición electrónica gratuita. Texto completo en www.eumed.net/libros/2007a/233/

Hermosilla Paul G. Vida Económica en https://billionbooksbaby.org/pdf-vidaeconomica.html

Ha-Joon Chang (2003). Patada a la escalera: La verdadera historia del libre comercio. Trabajo presentado en la conferencia sobre Globalization and the Myth of Free Trade, celebrada en la New School University de Nueva York. Facultad de Ciencias Económicas y Ciencias Políticas. Universidad de Cambridge.

Instituto de Investigaciones Económicas y Sociales (IIES) (2016). Observatorio Económico y de Emprendimiento (OEE); Universidad Nacional Autónoma de Honduras (UNAH), Fuerza Laboral en Honduras, Desempeño 2015 y Perspectivas 2016. Tegucigalpa.

Instituto Nacional de Estadísticas (INE) (2013). XVII Censo Nacional de Población y Vivienda. Gráfico No.3, Tegucigalpa.

Keynes, John Maynard (2007). Teoría general del empleo, el interés y el dinero. Resumen de Pérez Enrri-2007, Macroeconomía, Cs. Económicas, UBA en: http://www.altillo.com/examenes/uba/economicas/cicloprofesional/macroeconomia/ macro2007resukeynes.asp

Kiyosaki Robert y Sharon L. Lechter (2008). Padre rico padre pobre. Spanish Edition.

Piketty Thomas (2015). El Capital en el Siglo XXI, 1ª Edición, $2^{\text {a }}$ reimpresión, Ciudad Autónoma de Buenos Aires. Fondo de Cultura Económica.

Pavón Julián (2012). CEPADE Muerte y Resurrección de Keynes. Universidad Politécnica de Madrid. Badar Kablar en www.youtube.es

Sabillón Carlos (2009). Riqueza para Todos. Editorial Guardabarrancos, Tegucigalpa, Honduras. 
Secretaría de Finanzas de Honduras, SEFIN (2016) Página Web www.sefin.gob.hn. Dirección General de Política Macrofiscal. Cifras Fiscales y Memoria Institucional. Serie 20052015

Secretaría de Finanzas de Honduras, SEFIN (2016), Cuadro de Indicadores de Riesgo del Gobierno Central.

Serrano Tobías José Evelio (2016). Programa de Teoría Económica de Postgrado. Cátedra de Teoría Económica. Universidad Tecnológica Centroamericana (UNITEC). Material Didáctico.

Williamson John (2003). No hay consenso. Reseña sobre el Consenso de Washington y sugerencias sobre los pasos a dar. Revista Finanzas \& Desarrollo.

"LA REVISTA INNOVARE NO SE HACE RESPONSABLE EN NINGÚN CASO DE LOS CONTENIDOS, DATOS, CONCLUSIONES U OPINIONES VERTIDAS EN LOS ARTÍCULOS PUBLICADOS, SIENDO ESTA RESPOSABILIDAD EXCLUSIVA DEL (DE LOS) AUTOR (AUTORES)" 